\title{
Variabilidade sazonal de constituintes químicos (triterpenos, flavonóides e polifenóis) das folhas de Maytenus aquifolium Mart. (Celastraceae)
}

\author{
Janete H.Yariwake ${ }^{1 *}$, Fernando M. Lanças ${ }^{1}$, Esmeraldo A. Cappelaro ${ }^{1}$, Eliane C. de \\ Vasconcelos $^{1}$, Luciana A. Tiberti ${ }^{1}$, Ana M.S. Pereira ${ }^{2}$, Suzelei de C. Franca ${ }^{2}$ \\ ${ }^{1}$ Instituto de Química de São Carlos, Universidade de São Paulo, Caixa Postal 780, 13560-970, \\ São Carlos, SP, Brasil, \\ ${ }^{2}$ Departamento de Biotecnologia, Universidade de Ribeirão Preto, Av. Costábile Romano 2201, 14096-380, \\ Ribeirão Preto, SP, Brasil
}

\begin{abstract}
RESUMO: Estudou-se a variabilidade química sazonal em Maytenus aquifolium Mart. (Celastraceae), conhecida como "espinheira santa”, em exemplares cultivados em Ribeirão Preto, SP, através da análise das substâncias presentes nas folhas, ao longo das quatro estações em dois anos. O teor de fenóis totais variou de 21,96 a 45,92 mg / g (expresso em mg ácido tânico / g folhas secas), de 55,88 a 93,20 mg triterpenos totais / g folhas secas, e de 1,349 a 3,859 mg flavonóides / g folhas secas. A amostra Primavera 92 apresentou o maior teor de flavonóides e fenóis totais, e a amostra Inverno 94 apresentou o maior teor de triterpenos.
\end{abstract}

Unitermos: Maytenus aquifolium, Celastraceae, flavonóides, triterpenos, fenóis totais, variabilidade sazonal.

\begin{abstract}
Seasonal variability of chemical compounds (triterpenes, flavonoids and polyphenols) from Maytenus aquifolium Mart (Celastraceae) leaves”. The chemical seasonal variability of Maytenus aquifolium Mart. (Celastraceae), a Brazilian medicinal plant known as "espinheira santa”, was investigated on specimens cultivated at Ribeirão Preto SP, Brazil, at the four seasons of two years. The content of total phenolics, flavonoids and triterpenes varied from 21.96 to $45.92 \mathrm{mg}$ total phenolics (expressed as tanic acid) / g dried leaves, 55.88 - $93.20 \mathrm{mg}$ total triterpenes / g dried leaves, and 1.349 - 3.859 mg flavonoid / g dried leaves. Sample Spring 92 had the higher content of flavonoids and total phenolics, while sample Winter 94 had the highest content of triterpenes.
\end{abstract}

Keywords: Maytenus aquifolium, Celastraceae, flavonoids, triterpenes, total phenolics, seasonal variability.

\section{INTRODUÇÃO}

Várias plantas são conhecidas no Brasil com o nome popular "espinheira santa", dentre elas Maytenus aquifolium Mart. e Maytenus ilicifolia Mart. ex Reiss. (Celastraceae). Estudos demonstraram a atividade antiúlcera gástrica em ratos e seres humanos, utilizando-se os extratos aquosos das folhas (infusões) (Carlini, 1988; Souza-Formigone et al., 1991). O estudo fitoquímico indicou a presença de flavonóides tetraglicosilados, Figura 1 (Leite et al., 2001; Sannomiya et al., 1998; Vilegas et al., 1999).

Há também relatos sobre outras substâncias polifenólicas (possivelmente taninos) nos extratos aquosos (Carlini, 1988). A análise dos flavonóides por cromatografia de camada delgada de alta performance (HPTLC) demonstrou que esta é uma técnica importante para a análise e controle de qualidade de Maytenus (droga vegetal e medicamentos fitoterápicos), por possibilitar a deteç̧ão de adulterações através do perfil cromatográfico (“impressão digital”) (Vilegas et al., 1998).

O estudo das folhas de Maytenus aquifolium por cromatografia gasosa acoplada à espectrometria de massas (CG-EM) demonstrou também a presença de diversos triterpenos (Cordeiro et al., 1999). A friedelina e o friedelan-3-ol são os triterpenos majoritários nas folhas de Maytenus aquifolium e de Maytenus ilicifolia, e assim foi proposto um método para a sua quantificação (Nossack et al., 2000), visando o estabelecimento de critérios gerais de qualidade para os medicamentos fitoterápicos contendo "espinheira santa" (Vilegas et al., 1994; Vilegas; Lanças, 1997).

Devido à crescente utilização da "espinheira santa", a coleta indiscriminada ameaça levar ao desaparecimento dos exemplares nativos, o que motivou estudos sobre o seu cultivo visando o seu aproveitamento econômico, inclusive como matéria-prima para a indústria de medicamentos fitoterápicos. Maytenus aquifolium e 
<smiles>[R]c1cc(-c2oc3cc(O)cc(O)c3c(=O)c2OC([R11])[Y10]([H])([H])[3H])ccc1O</smiles>

$\mathrm{R}$

OH Quercetina 3-O- $\alpha$-L-ramnopiranosil $(1 \rightarrow 6)$ - $O$-[ $[\beta$-D-glucopiranosil $(1 \rightarrow 3)$ $O$ - $\alpha$-L-ramnopiranosil $(1 \rightarrow 2)$ - $O$ - $\beta$-D-galactopiranosídeo

$2 \mathrm{H}$ Canferol 3-O- $\alpha$-L-ramnopiranosil $(1 \rightarrow 6)$ - $O$-[ $\beta$-D-glucopiranosil $\quad(1 \rightarrow 3)$ $O$ - $\alpha$-L-ramnopiranosil $(1 \rightarrow 2)-O$ - $\beta$-D-galactopiranosídeo

Figura 1. Estrutura dos flavonóides de Maytenus aquifolium

Maytenus ilicifolia são nativas da região sudeste do Brasil, mas a primeira é a melhor adaptada a climas mais quentes como por exemplo na região de Ribeirão Preto, SP, que localiza-se acima do Trópico de Capricórnio (Pereira et al., 1995).

Para propor critérios de padronização de medicamentos fitoterápicos é imprescindível conhecer a variabilidade química da espécie vegetal utilizada como matéria-prima. No caso do cultivo de Maytenus aquifolium, estudou-se o efeito de diferentes procedimentos de fertilização sobre algumas características morfológicas e na produção de triterpenos e de fenóis totais (Pereira et al., 1995). Observou-se a influência da fertilização na produção de biomassa total, mas não foram constatadas alterações significativas na concentração de triterpenos e de fenóis totais. Não há relatos na literatura, porém, sobre as flutuações sazonais na composição química de Maytenus aquifolium. Deve-se considerar que a variabilidade química sazonal pode afetar o teor de princípios ativos de plantas medicinais cultivadas, sendo por isso um importante fator a ser estudado para o estabelecimento de critérios de qualidade para plantas medicinais cultivadas (List; Schmidt, 1989).

Este trabalho apresenta os resultados da análise quantitativa de flavonóides, fenóis totais e triterpenos, em folhas obtidas de exemplares cultivados em Ribeirão Preto, SP, de Maytenus aquifolium, cobrindo o intervalo de dois anos de coleta. Neste trabalho é também relatado o procedimento analítico desenvolvido para a análise quantitativa por cromatografia líquida de alta eficiência (CLAE) dos flavonóides de Maytenus aquifolium, sem a necessidade de sua hidrólise.

\section{MATERIAL E MÉTODOS}

\section{Material vegetal}

As amostras de Maytenus aquifolium foram obtidas de espécimens cultivados seguindo práticas de agricultura orgânica, em Ribeirão Preto, SP. As folhas foram coletadas no final de cada estação, do inverno de 1992 ao outono de 1993 e do inverno de 1994 ao outono de 1995. As amostras foram secas $\left(40{ }^{\circ} \mathrm{C}\right.$, ventilação forçada, 3 dias $)$, pulverizadas $(0.5-1.0 \mathrm{~mm})$ e armazenadas em frascos fechados e protegidos da luz. As amostras foram secas novamente a $30-40{ }^{\circ} \mathrm{C}$ por 1 hora imediatamente antes de serem submetidas ao procedimento de extração.

\section{Reagentes e padrões analíticos}

Utilizou-se solventes orgânicos de grau analítico (Merck, Darmstadt, Germany) ou HPLC (Mallincrodt, Kentucky, U.S.A.) e água purificada em um sistema MilliQ (Waters). Os padrões dos flavonóides rutina, quercetina e canferol foram adquiridos da Sigma (St. Louis, U.S.A.) e o padrão de ácido tânico foi adquirido da Vetec (Rio de Janeiro, Brasil). O padrão de friedelina foi isolado e purificado a partir do extrato hexânico das folhas de Maytenus aquifolium, conforme previamente descrito (Nossack et al., 2000).

\section{Extração dos flavonóides}

Folhas pulverizadas (1 g) foram extraídas com $10 \mathrm{~mL}$ de metanol: água $(1: 1, \mathrm{v} / \mathrm{v})$ a $50{ }^{\circ} \mathrm{C}$, usando agitador magnético por 30 min. Após filtração, o extrato 
hidrometanólico foi submetido a partição com $8 \mathrm{~mL}$ de uma mistura clorofórmio-metanol 5:3 (v/v); as fases foram separadas e a fase hidrometanólica foi filtrada e diretamente analisada por CLAE.

\section{Análise dos flavonóides por CLAE}

Utilizou-se um sistema modular Shimadzu composto por duas bombas LC-10AD, detector UV SPD M10A de arranjo de diodos, equipado com válvula de injeção Rheodyne com loop de $10 \mu \mathrm{L}$ e sistema LC WorkStation Class LC10 para processamento dos dados. Coluna: RP-8 (250 mm x $4.6 \mathrm{~mm}$ x $5 \mu \mathrm{m}$ ), Supelco; temperatura do forno $=33{ }^{\circ} \mathrm{C}$. Como fase móvel, utilizou-se ácido fórmico $2 \%$ em água (v/v, eluente A) e acetonitrila (eluente B); antes do início da análise, equilibrou-se o sistema com uma mistura de $85 \%$ A: $15 \%$ B. Programação do gradiente: linear, 15 - 80\% B em 20 min à vazão de 1 mL/min. Detecção: $350 \mathrm{~nm}$.

Análise quantitativa: utilizou-se o método do padrão externo, utilizando soluções de quercetina (0,05 - 0,50 $\mathrm{mg} / \mathrm{mL})$ e de canferol $(0,05-0,40 \mathrm{mg} / \mathrm{mL})$ preparadas em acetonitrila - água 1:1 (v/v). Os valores de concentração não foram corrigidos, pois o grau de pureza dos padrões comerciais utiizados não é declarado pelos fabricantes.O limite de detecção foi estabelecido através da injeção de soluções-padrão de quercetina e de canferol, até chegar-se a um pico cromatográfico de razão sinal-ruído de 3:1, nas condições cromatográficas descritas. Todas as análises foram feitas em triplicata.

\section{Análise dos triterpenos por CG}

O preparo de amostras (extração e clean-up) para a análise quantitativa dos triterpenos de Maytenus aquifolium foi anteriormente otimizado e descrito em detalhes (Nossack et al., 2000; Vasconcelos et al., 2000). As análises foram feitas em triplicata. As análises por CG foram feitas em um cromatógrafo HP 5890 Série II (Hewlett Packard), equipado com detector de ionização de chama e integrador HP 3396 A. Parâmetros da análise: split 1:33 $(1 \mu \mathrm{L})$, gás de arraste, hidrogênio $(65 \mathrm{~cm} / \mathrm{s})$, coluna LM-5 (L\&M), com fase estacionária 95\% metil, $5 \%$ fenil-polisiloxano cross-linked com $38 \mathrm{~m}$ x $0.25 \mathrm{~mm}$ d.i. e espessura do filme de $0,35 \mu \mathrm{m}$. Programação da temperatura de análise: $\mathrm{T}_{\text {inicial }}=280{ }^{\circ} \mathrm{C}$ por $22 \mathrm{~min}, 280$ $310^{\circ} \mathrm{C}\left(12^{\circ} \mathrm{C} / \mathrm{min}\right), \mathrm{T}_{\text {final }}=310^{\circ} \mathrm{C}$ por $1 \mathrm{~min}$. Temperatura do injetor $280{ }^{\circ} \mathrm{C}$ e do detector $320^{\circ} \mathrm{C}$.

\section{Análise dos fenóis totais}

As folhas pulverizadas (200 mg) foram extraídas com metanol $50 \%$ em ebulição ( $10 \mathrm{~mL}, 5$ vezes). Os extratos hidrometanólicos foram filtrados, reunidos e ajustou-se o volume a $100 \mathrm{~mL}$ com metanol 50\%. Alíquotas (0,2, 0,3, 0,4 e $0,5 \mathrm{~mL}$ ) foram analisadas utilizando-se o método de Folin-Denis (Waterman; Mole, 1994). A extração e a análise espectrofotométrica foram feitas em triplicata, e os resultados foram calculados em mg ácido tânico /g material vegetal seco.

\section{Tratamento estatístico dos dados}

Utilizou-se o software Microcal Origin 5.0 (Microcal Software Inc.) para o cálculo das equações de regressão linear, teste $t$ de Student e teste ANOVA.

\section{RESULTADOS}

As Tabelas 1 a 3 apresentam os resultados quantitativos para cada classe de substâncias (flavonóides, fenóis totais e triterpenos). O seu teor variou no intervalo de: 1,349 a 3,859 mg flavonóides / $\mathrm{g}$ folhas secas; 21,96 a 45,92 mg fenóis totais (expresso em ácido tânico) / g folhas secas e 55,88 a 93,20 mg triterpenos totais / $\mathrm{g}$ folhas secas. A amostra "primavera 92" apresentou o maior teor de flavonóides e fenóis totais, enquanto que o maior teor de triterpenos foi encontrado na amostra "inverno 94".

O perfil cromatográfico dos flavonóides de Maytenus aquifolium é relativamente simples (Figura 2), apresentando dois picos majoritários. A quantificação dos flavonóides totais foi feita usando-se quercetina e canferol como padrões, respectivamente para os picos 1 e 2 (Tabela 1), devido à inexistência de padrões comerciais dos flavonóides de M. aquifolium (derivados glicosilados de quercetina e de canferol, Figura 1)

\section{DISCUSSÃO}

Para a quantificação dos flavonóides totais, foi necessário inicialmente desenvolver e validar o procedimento de análise por CLAE. Utilizou-se uma coluna com fase estacionária do tipo $\mathrm{C}-8$, pois testes preliminares indicaram menor tempo de análise, em comparação com o uso de fase tipo C-18. O uso de ácido fórmico na fase móvel mostrou-se necessário para a boa resolução e simetria dos picos cromatográficos. Utilizando-se as condições cromatográficas otimizadas, descritas neste trabalho, constatou-se a grande semelhança no perfil cromatográfico de todas as amostras, exceto pela ausência de uma substância minoritária nas amostras "inverno 1992" e "inverno 1994" (ombro do pico 1, Figura 2). Este pico corresponde a um derivado glicosilado do canferol, anteriormente identificado através de análises por eletroforese capilar (CE) e cromatografia líquida - espectrometria de massas (LC/MS), em amostras de Maytenus aquifolium (Tiberti et al., 1998).

Como os demais métodos analíticos utilizados (determinação de triterpenos totais e de fenóis totais) foram previamente padronizados e otimizados, as variações quantitativas observadas estão diretamente correlacionadas às flutuações na composição química das amostras. Assim, os resultados apresentados nas Tabelas 1 a 3 são discutidos a seguir com a ajuda de testes 
Tabela 1. Teor de flavonóides em folhas de Maytenus aquifolium, determinado por CLAE-UV. Resultados expressos em $\mu$ mol flavonóide / g folhas secas*

\begin{tabular}{lccc}
\hline \multicolumn{1}{c}{ Amostra } & pico $1^{+}$ & pico $2^{++}$ & Flavonóides totais $^{\#}(1+2)$ \\
\hline Inverno 1992 & $1,19 \pm 0,01$ & $1,76 \pm 0,01$ & $2,95 \pm 0,01$ \\
Primavera 1992 & $1,35 \pm 0,06$ & $2,51 \pm 0,07$ & $3,86 \pm 0,03$ \\
Verão 1992 & $0,64 \pm 0,04$ & $1,14 \pm 0,09$ & $1,78 \pm 0,12$ \\
Outono 1993 & $1,21 \pm 0,06$ & $2,26 \pm 0,08$ & $3,47 \pm 0,14$ \\
& & & \\
Inverno 1994 & $0,51 \pm 0,03$ & $0,84 \pm 0,04$ & $1,35 \pm 0,04$ \\
Primavera 1994 & $0,94 \pm 0,02$ & $1,55 \pm 0,03$ & $2,49 \pm 0,04^{\mathrm{a}}$ \\
Verão 1994 & $0,87 \pm 0,02$ & $1,62 \pm 0,05$ & $2,49 \pm 0,06^{\mathrm{a}}$ \\
Outono 1995 & $1,11 \pm 0,03$ & $1,83 \pm 0,05$ & $2,95 \pm 0,07$ \\
\hline
\end{tabular}

* média \pm desvio-padrão $(\mathrm{n}=6)$

${ }^{+}$valores calculados como quercetina; limite de detecção $=0,3 \mu \mathrm{g} / \mathrm{mL}$

${ }^{++}$valores calculados como canferol; limite de detecção $=0,2 \mu \mathrm{g} / \mathrm{mL}$

\# a comparação estatística dos resultados foi realizada apenas com os valores de flavonóides totais

${ }^{a}$ médias não diferindo estatisticamente entre si (teste $t, \mathrm{p}=0,05$ )

Tabela 2. Teor de fenóis totais em folhas de Maytenus aquifolium, determinado pelo método de Folin-Denis. Resultados expressos em mg ácido tânico / g folhas secas*

\begin{tabular}{lc}
\hline Amostra & Fenóis totais \\
\hline Inverno 1992 & $42,93 \pm 2,56^{\mathrm{a}, \mathrm{e}}$ \\
Primavera 1992 & $45,92 \pm 3,14^{\mathrm{e}}$ \\
Verão 1992 & $41,43 \pm 1,74^{\mathrm{a}, \mathrm{e}, \mathrm{b}}$ \\
Outono 1993 & $40,67 \pm 0,74^{\mathrm{b}, \mathrm{e}}$ \\
& \\
Inverno 1994 & $21,96 \pm 0,28$ \\
Primavera 1994 & $39,00 \pm 2,03^{\mathrm{c}}$ \\
Verão 1994 & $31,83 \pm 2,03^{\mathrm{d}}$ \\
Outono 1995 & $35,63 \pm 0,76^{\mathrm{c}, \mathrm{d}}$ \\
\hline
\end{tabular}

* média \pm desvio-padrão $(\mathrm{n}=3)$

a, b, c, d os pares de resultados com a mesma letra em sobrescrito não diferem estatisticamente entre si (teste $t, \mathrm{p}=0,05$ )

${ }^{\mathrm{e}}$ médias não diferindo estatisticamente entre si (ANOVA, $\left.\mathrm{p}=0,05\right)$

estatísticos (teste- $t$ de Student eANOVA). As comparações estatísticas foram feitas entre todos os resultados das amostras dos dois períodos sazonais anuais.

Observou-se correlação do teor de friedelan3-ol e de friedelina, presentes em todas as amostras na proporção de 5:1 a 4:1. Este padrão foi também observado em amostras comerciais de Maytenus; como regra geral, este é um padrão característico ('impressão digital') de amostras autênticas de Maytenus aquifolium e Maytenus ilicifolia (Nossack et al., 2000; Vilegas; Lanças, 1997; Vilegas et al., 1998). A determinação do teor de triterpenos totais, portanto, é um critério mais simples do que a análise do teor individual dos triterpenos friedelina e friedelan-3-ol. A variação no teor de triterpenos indica uma correlação inversa entre a produção de triterpenos e de fenóis totais, como pode ser observado comparando-se os gráficos das Figuras $3 \mathbf{a}-\mathbf{c}$.

Observou-se também correlação na flutuação do teor de fenóis totais e de flavonóides, com exceção da amostra "verão 92”. Este resultado é coerente com o fato de que os flavonóides com grupos fenólicos livres também reagem com o reagente de Folin-Denis (Waterman; Mole, 1994). A representação gráfica mostra que, como tendência geral, tanto os flavonóides totais (Figura 3a) como os fenóis totais (Figura $3 \mathrm{~b}$ ) são produzidos em menor concentração em estações com fotoperíodos bem definidos: inverno (dias curtos e noites longas) e verão (dias longos e noites curtas), enquanto que a maior 
Tabela 3. Teor de triterpenos em folhas de Maytenus aquifolium, determinado por cromatografia gasosa. Resultados expressos em mg triterpenos / $g$ folhas secas ${ }^{1}$

\begin{tabular}{lccc}
\hline Amostra & Friedelan-3-ol & Friedelina & $\begin{array}{c}\text { Triterpenos totais }^{\#} \\
\text { (friedelan-3-ol + friedelir }\end{array}$ \\
\hline Inverno 1992 & $72,22 \pm 6,28$ & $16,41 \pm 1,34$ & $88,63 \pm 7,58^{\mathrm{a}}$ \\
Primavera 1992 & $44,80 \pm 2,08$ & $11,08 \pm 0,51$ & $55,88 \pm 2,59$ \\
Verão 1992 & $68,06 \pm 1,77$ & $14,16 \pm 0,49$ & $82,22 \pm 2,25^{\mathrm{a}}$ \\
Outono 1993 & $61,77 \pm 0,71$ & $14,05 \pm 0,45$ & $75,82 \pm 0,26$ \\
& & & $93,20 \pm 5,85$ \\
Inverno 1994 & $71,22 \pm 4,47$ & $21,98 \pm 1,37$ & $67,23 \pm 1,72^{\mathrm{b}}$ \\
Primavera 1994 & $54,87 \pm 1,23$ & $12,36 \pm 0,49$ & $78,73 \pm 1,04$ \\
Verão 1994 & $64,51 \pm 0,72$ & $14,22 \pm 0,34$ & $68,31 \pm 3,68^{\mathrm{b}}$ \\
Outono 1995 & $55,76 \pm 2,89$ & $12,55 \pm 0,78$ & \\
\hline
\end{tabular}

${ }^{1}$ média \pm desvio-padrão( $\left.\mathrm{n}=3\right)$

* a comparação estatística dos resultados foi realizada apenas com os valores de triterpenos totais

${ }^{\mathrm{a}, \mathrm{b}}$ os pares de resultados com a mesma letra em sobrescrito não diferem estatisticamente entre si (teste $t, \mathrm{p}=0,05$ )

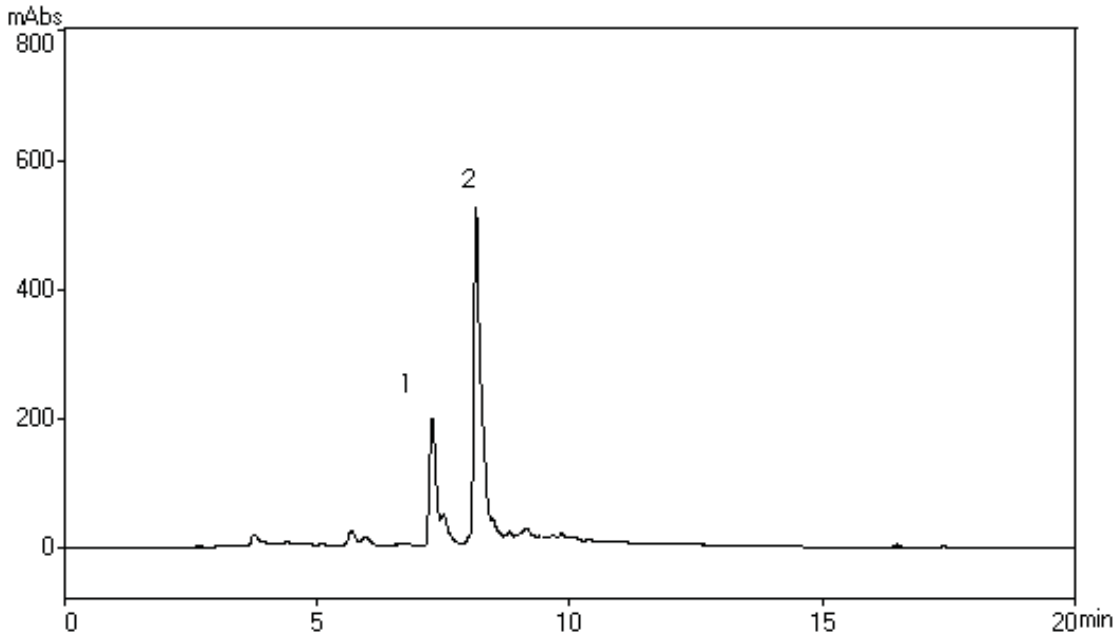

Figura 2. Cromatograma típico (CLAE, coluna C-8) do extrato hidro-metanólico das folhas de Maytenus aquifolium. Identificação dos picos: 1, derivado de quercetina; 2, derivado de canferol. Condições cromatográficas: vide Materiais e Métodos

produção destes metabólitos está correlacionada com as estações que apresentam diferença menos nítida na duração dos dias e noites (primavera e outono). Esta tendência é coerente com o papel fotoprotetor dos fenóis, inclusive os flavonóides, na defesa das plantas (Harborne, 1994).

Em conclusão, constatou-se variações sazonais significativas na composição química das folhas de Maytenus aquifolium. Estes resultados indicam que a utilização de Maytenus aquifolium como matéria-prima de medicamentos fitoterápicos requer a padronização do teor de flavonóides e de triterpenos. A legislação brasileira também indica a importância dos estudos analíticos, para a adequada definição de parâmetros de qualidade dos medicamentos fitoterápicos (Petrovick et al., 1999).

\section{AGRADECIMENTOS}

À CAPES, CNPq e FAPESP pelo auxílio financeiro e bolsas. Os autores também agradecem ao Prof. Dr. Wagner Vilegas (IQ, UNESP) pelas amostras de referência dos flavonóides de M. aquifolium. 


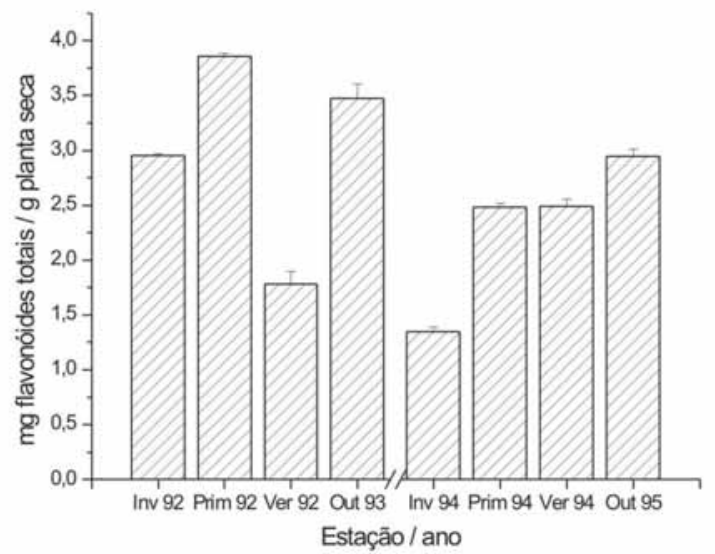

(a)

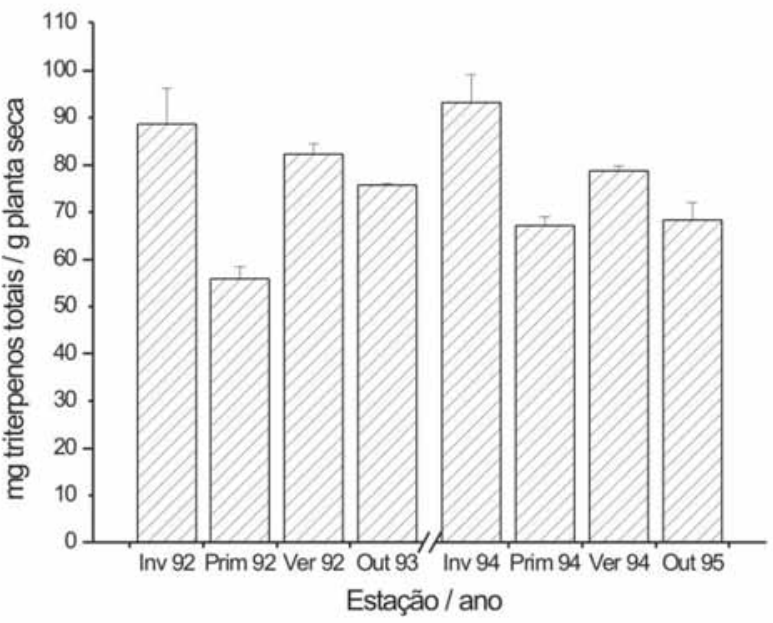

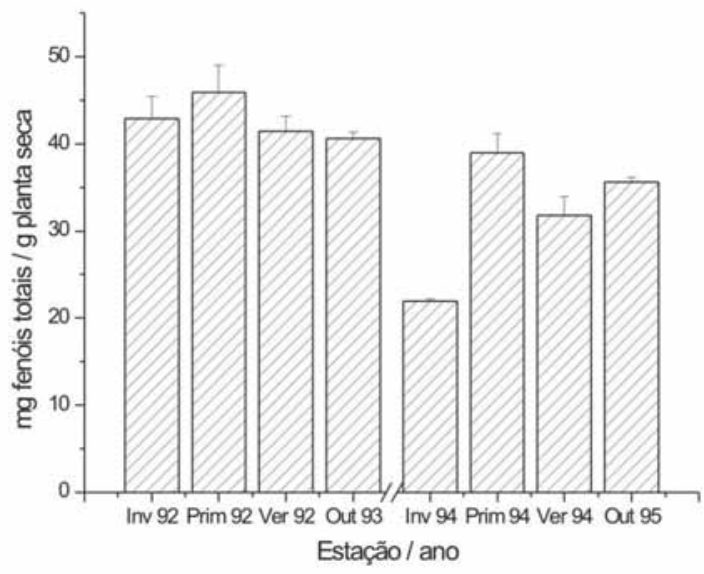

(b)

\section{(c)}

Figura 3. Representação gráfica da variação de substâncias das folhas de Maytenus aquifolium em função da estação do ano: (a) flavonóides totais; (b) fenóis totais; (c) triterpenos totais

\section{REFERÊNCIAS}

Carlini ELA 1988. Estudo da ação anti úlcera gástrica de plantas brasileiras: Maytenus ilicifolia (Espinheira santa e outras). Brasília: CEME/AFIP.

Cordeiro PJM, Vilegas JHY, Lanças FM 1999. HRGC-MS analysis of terpenoids from Maytenus ilicifolia and Maytenus aquifolium ("espinheira santa”). J Braz Chem Soc 10: 523-526.
Harborne JB 1994, The flavonoids - advances in research since 1986. London: Chapman \& Hall.

Leite JPV, Rastrelli L, Romussi G, Oliveira AB, Vilegas JHY, Vilegas W, Pizza C 2001. Isolation and HPLC quantitative analysis of flavonoid glycosides from Brazilian beverages (Maytenus ilicifolia and $M$. aquifolium). J Agric Food Chem 49: 3796-3801.

List PH, Schmidt PC 1989. Phytopharmaceutical technology. London: Heyden \& Son. 
Nossack AC, Vasconcelos EC, Vilegas JHY, Lanças FM, Roque NF 2000. Quantitative analysis of triterpenes friedelin and friedelan-3-ol in Maytenus aquifolium by HRGC and HT-CGC. Phytochem Anal 11: 243-246.

Pereira AMS, Menezes JrA, França SC, Vilegas JHY, Cordeiro PJM, Lanças FM 1995. Effect of fertilization on morphologic characteristics and secondary metabolites of Maytenus aquifolium Mart. J Herbs, Spices Med Plants 3: 43-50.

Petrovick PR, Marques LC, de Paula IC 1999. New rules for phytopharmaceutical drug registration in Brazil. $J$ Ethnopharmacol 66: 51-55.

Sannomiya M, Vilegas W, Rastrelli L, Pizza C 1998. A flavonoid glycoside from Maytenus aquifolium. Phytochemistry 49: 237-239.

Souza-Formigone MLO, Oliveira MGM, Monteiro MG, Silveira Filho NG, Braz S, Carlini EA 1991. Antiulcerogenic effects of two Maytenus species in laboratory animals. J Ethnopharmacol 34: 21-27.

Tiberti LA, Vilegas JHY, Lanças FM, Rossi M, Celli N, Rotilio D 1998. LC and CE fingerprint analysis of flavonoids in medicinal plants. In: International Symposium on Capillary Chromatography, 20., 1998. Riva del Garda. Proceedings. Kortrjk: IOPMS, 1998. 1 CD-ROM.

Vasconcelos EC, Vilegas JHY, Lanças FM 2000. Comparison among extraction and clean-up methods for analysis of friedelin and friedelan-3-ol from leaves of Maytenus aquifolium Martius (Celastraceae). Phytochem Anal 11: 247-250.

Vilegas JHY, Lanças FM 1997. Detecção de adulterações em amostras comerciais de "espinheira santa" por cromatografia gasosa de alta resolução (HRGC). Rev Ciênc Farm 18: 241-248.

Vilegas JHY, Lanças FM, Cervi AC 1994. High resolution gas chromatography analysis of "espinheira santa" (Maytenus ilicifolia and M. aquifolium): analysis of crude drug adulterations. Phytother Res 8: 241-244

Vilegas JHY, Lanças FM, Wauters J.N, Angenot L 1998. Characterization of adulterations of "espinheira santa” (Maytenus ilicifolia M. and M. aquifolium M., Celastraceae) hydroalcoholic extracts with Sorocea bomplandii (Moraceae) by HPTLC. Phytochem Anal 9: 263-266.

Vilegas W, Sanommiya M., Rastrelli L, Pizza C 1999. Isolation and structure elucidation of two new flavonoid glycosides from the infusion of Maytenus aquifolium leaves. Evaluation of the antiulcer activity of the infusion. J Agric Food Chem 47: 403-406.

Waterman PG, Mole S 1994. Analysis of phenolic plant metabolites. Oxford: Blackwell.

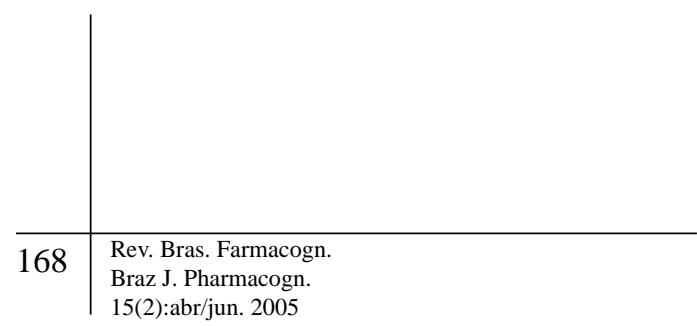

